\title{
Pediatric idiopathic hypercalciuria with bilateral nephrolithiasis and hypertensive urgency
}

\author{
Natharina Yolanda* and Irene*
}

\section{ABSTRACT}

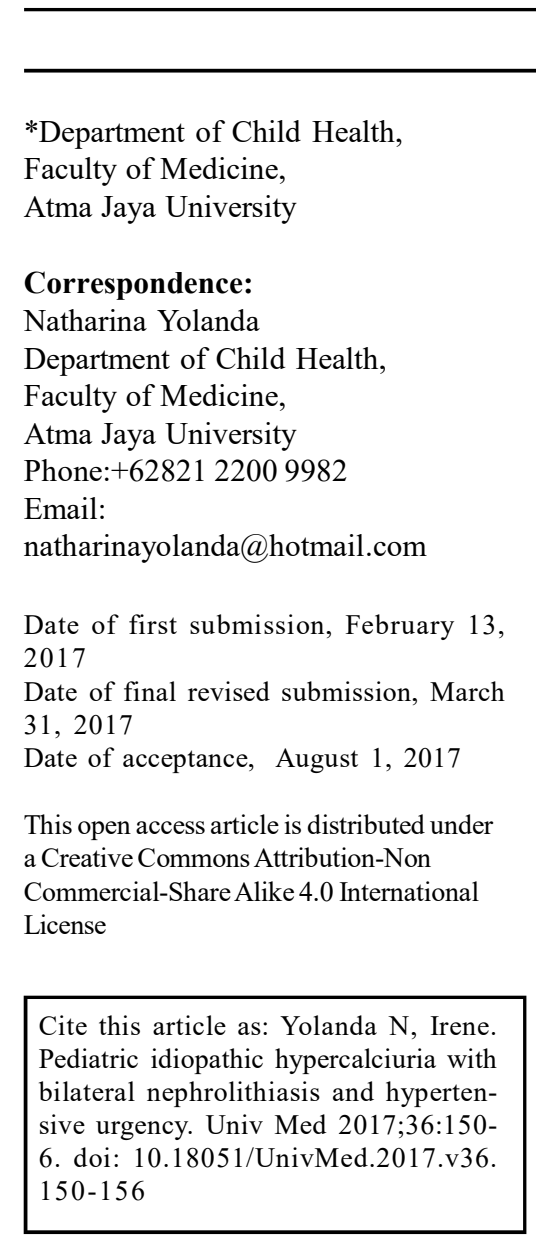

*Department of Child Health, Faculty of Medicine,

Correspondence:

Natharina Yolanda Department of Child Health, Faculty of Medicine,

Atma Jaya University

Email:

Date of first submission, February 13, Date of final revised submission, March 31,2017

This open access article is distributed under a Creative Commons Attribution-Non Commercial-Share Alike 4.0 International

this article as: Yolanda $\mathrm{N}$, Irene. Pediatric idiopathic hypercalciuria with sive urgency. Univ Med 2017;36:150

\section{1} sources. Metabolic evaluation is mandatory in pediatric renal stone patients and hypercalciuria is an important etiology to be evaluated. Ignorance of the underlying metabolic abnormality or failure in its identification in pediatric urolithiasis could lead to catastrophic long-term effects.

Keywords: Idiopathic hypercalciuria, pediatric nephrolithiasis, renal stones

\section{BACKGROUND}

Pediatric nephrolithiasis is an important cause of morbidity worldwide. Even with low prevalence, nephrolithiasis can cause detrimental longterm effects on kidney function. Idiopathic hypercalciuria is the most common cause of renal stones in children. This case report presents a pediatric patient with bilateral nephrolithiasis and hydronephrosis associated with underlying idiopathic hypercalciuria.

\section{CASE DESCRIPTION}

We report on a 17-month-old boy presenting with two days of high fever, irritability, nausea, and poor feeding. The patient had been diagnosed with bilateral nephrolithiasis at the age of 6 months. On examination, the patient was febrile and hypertensive. Laboratory evaluation revealed anemia, leukocytosis, normokalemia, normocalcemia, and normal glomerular filtration rate. Blood gases were within normal limits. Urinalysis revealed $\mathrm{pH}$ 5.0, hematuria, pyuria, and bacteriuria. Urine culture was positive for Proteus mirabilis and Enterobacter gergoviae. Electrolyte analysis from urine samples revealed hypercalciuria. Ultrasonography found stones at the left ureteropelvic junction with corresponding grade I hydronephrosis and stones in the right lower calyx without hydronephrosis. The patient was treated with antibiotics, an antihypertensive agent, thiazide diuretics, and was referred to a pediatric urologist.

\section{CONCLUSION}

This case can be considered as idiopathic. Most children with renal stones have metabolic risk factors including enteric, endocrinologic, or rena 


\section{INTRODUCTION}

Pediatric nephrolithiasis is an important cause of morbidity worldwide, with detrimental long-term effects on kidney function. Recent data provide evidence that the incidence of nephrolithiasis in children is rising. In the United States, pediatric nephrolithiasis accounts for 1 per 1,000 to 7,600 pediatric hospital admissions. ${ }^{(1-3)}$ Data from Indonesia are not available. Approximately $50 \%$ of these patients have an onset around 5 years of age. One characteristic feature of pediatric stone disease is the frequency of an underlying metabolic etiology. It has been reported that $50 \%$ of children aged $<10$ years with stone disease have an underlying metabolic disorder. ${ }^{(4)}$ Underlying metabolic risk factors include enteric, endocrinologic, and renal sources. Hypercalciuria is the most common cause of stones in children, representing up to $50 \%$ of metabolic risk factors identified, followed by hypocitraturia. In children with nephrolithiasis or nephrocalcinosis, hypercalciuria is found in 28 $79 \%$ of cases. Most causes of hypercalciuria are idiopathic, both sporadic and familial. ${ }^{(2)}$

Hypercalciuria with nephrolithiasis is associated with a wide variety of symptoms. The most common symptoms are abdominal pain (53$75 \%$ ), gross hematuria (14-33\%), and dysuria (15\%), although $15 \%$ of cases present as an asymptomatic, incidental radiologic finding. ${ }^{(3,4)}$ Urinary tract infection (UTI) is also a common associated symptom, affecting $8-45 \%$ children with nephrolithiasis. ${ }^{(1)}$ In infants, symptoms of nephrolithiasis may not be specific (irritability, nausea, fever, hematuria) and UTI may be the presenting sign up to $75 \%$ of the time. It is particularly important to recognize the combination of obstructing stone and UTI as this clinical scenario can quickly lead to sepsis. ${ }^{(1)}$ Although urolithiasis may affect children of all ages, little information is available regarding preschool children. ${ }^{(5)}$

The aim of this case report was to present a pediatric patient with bilateral nephrolithiasis and hydronephrosis associated with underlying idiopathic hypercalciuria and to emphasize the importance of looking deeper than the stone to find the underlying abnormalities.

\section{CASE REPORT}

A 17-month-old boy presented with two days of fever, with a maximum of $40.4^{\circ} \mathrm{C}$. Additional symptoms were irritability, nausea, and poor feeding. Previously, the patient had been diagnosed with bilateral nephrolithiasis at the age of 6 months, the initial presentation being hematuria and high fever. At that time, urinalysis revealed 12-15 white blood cells (WBC) per high power field, $8-10$ red blood cells (RBC) per high power field, and crystals. Leukocyte esterase and nitrite tests were negative. Abdominal ultrasonography (USG) revealed multiple renal stones in the left lower calyx $(0.3,0.6$ and $0.7 \mathrm{~cm}$ in diameter) without hydronephrosis and multiple renal stones in the right lower calyx $(0.4$ and $0.5 \mathrm{~cm}$ in diameter) without hydronephrosis. Two months after the initial symptoms, the patient spontaneously passed two white stones, each 0.3 $\mathrm{cm}$ in diameter. Stone analysis was positive for calcium, oxalate, phosphate, and cystine. There had been two episodes of urinary tract infection since the diagnosis of nephrolithiasis was made. Each episode was treated with outpatient antibiotics. Family history of stone disease, renal dysfunction, gout, or arthritis were denied.

On examination, the patient was irritable, with blood pressure of $160 / 90 \mathrm{mmHg}\left(>\mathrm{P}_{99}+5\right.$ $\mathrm{mmHg}$ ), heart rate of 128 beats per minute, respiratory rate of 36 times per minute, and temperature of $38.4^{\circ} \mathrm{C}$. Conjunctivae, skin, and the oral mucous membrane were pale. There was no tenderness at the costovertebral angle. The patient has no phimosis and was circumcised. Laboratory evaluation revealed microcytic hypochromic anemia consistent with chronic disease (hemoglobin level $8.5 \mathrm{~g} / \mathrm{dL}$, hematocrit level 28\%, MCV 64 fl, MCH 19.2 pg, MCHC 25 $\mathrm{g} / \mathrm{dL}$, reticulocyte $1.4 \%$, ferritin $26.4 \mathrm{ng} / \mathrm{mL}$, and serum iron $49 \mathrm{mg} / \mathrm{dL}$ ), leukocytosis (WBC $24,700 / \mathrm{mL}$ with band predominance), 
normokalemia (serum potassium $3.4 \mathrm{mmol} / \mathrm{L}$ ), normocalcemia (serum ionized calcium $1.3 \mathrm{mmol} /$ L), and normal glomerular filtration rate (GFR $\left.83 \mathrm{~mL} / \mathrm{min} / 1.73 \mathrm{~m}^{2}\right)$. Urinalysis revealed $\mathrm{pH} 5.0$, pyuria (60-70 WBC per high power field), and Gram negative rods. Leukocyte esterase and nitrite were negative. Urine culture from bladder catheterization was positive for Proteus mirabilis $(>100,000$ colony-forming units $(\mathrm{CFU}) / \mathrm{mL})$ and Enterobacter gergoviae (1,000 - 10,000 CFU/ $\mathrm{mL}$ ). Electrolyte analysis from 24-hour urine samples revealed hypercalciuria (urinary calcium $400 \mathrm{mg} / \mathrm{kg} / \mathrm{day}$, ten times the normal value). Repeat US found stones of $7 \mathrm{~mm} \times 3 \mathrm{~mm}$ in the left uretheropelvic junction (UPJ) with corresponding grade I hydronephrosis and stones of $2 \mathrm{~mm}$ in diameter in the right lower calyx without hydronephrosis.

The patient was diagnosed as having urinary tract infection with predisposition of bilateral nephrolithiasis caused by idiopathic hypercalciuria. Complications were hypertensive urgency, hydronephrosis, and anemia of chronic disease. The hypertensive crisis was treated initially with oral nifedipine and controlled with oral captopril. UTI was treated with intravenous cefotaxime, with the fever subsiding after 72 hours, when the intravenous antibiotic was shifted to oral cefixime. The antibiotics were given for a total of 10 days. The patient was given prophylactic antibiotics and then referred to a pediatric urologist for further management.

\section{DISCUSSION}

Epidemiologic studies have shown a progressive increase in the incidence of pediatric urolithiasis (PU) worldwide over the few decades. ${ }^{(6)} \mathrm{PU}$ affects children of all ages and seems to primarily appear in the first years of life, which might be due to the fact that it is frequently based on tubulopathies or inborn errors of metabolism. ${ }^{(7)}$ The true burden in Indonesia is not known due to limited data; to date, here have been few case reports on this topic. The present case is important because there have been not many similar cases reported in Indonesia; this report is intended to be a trigger for doctors to increase their awareness and knowledge regarding the need of an appropriate search for the underlying metabolic abnormalities and their treatment in pediatric patients with urolithiasis.

Pediatric urinary stone disease is a relatively rare pathology, with overall incidence of $1-2 \% .{ }^{(8)}$ Predisposing causes can be recognized in $>75 \%$ of children and adolescents with urolithiasis and even the first stone episode deserves a comprehensive examination. Approximately $40 \%$ of children with urolithiasis have a positive family history of renal stones and most of them have a metabolic background. ${ }^{(9)}$ This disease is associated with considerable morbidity with reported recurrence rates ranging from $6.5-44 \%$ and appears to be highest in children with metabolic abnormalities. $^{(8)}$

A history of high fever and non-specific symptoms, pyuria, hematuria, and positive urine culture in this young child previously diagnosed with nephrolithiasis is consistent with UTI, specifically pyelonephritis. Proteus mirabilis is one of the most common urinary pathogen. The patient's history of recurrent urinary infections indicates the need for an attempt to identify predisposing factors. Some risk factors can be eliminated for this patient, which are female gender, uncircumcised male, toilet-training, and constipation. The mechanism of UTI in patients with hypercalciuria is due to the damage of the epithelium caused by microcrystal aggregates formed secondary to hypercalciuria, which provides a nidus for bacterial sequestration. ${ }^{(5)}$

In this patient, nephrolithiasis was identified by US to be a risk factor. Based on a previous study, nephrolithiasis is more likely to be found in young children whereas ureteral stones are more likely to be found in older children. Most pediatric renal stones are found in the upper urinary tract. ${ }^{(10)}$ Several other studies noted a slight predominance of boys in pediatric urolithiasis, with a boy:girl ratio of $1.3: 1.0$. If the stone is identified in the urinary tract but not passed in the urine, an evaluation of anatomic and metabolic 
abnormalities is recommended. Younger children are more likely to have an underlying metabolic risk factor. Based on several studies, the most common metabolic disorders are hypercalciuria and hyperuricosuria. However, there is no significant difference in frequency of metabolic abnormalities between patients aged $<3$ years and $\geq 3$ years. The risk factors for urolithiasis depend on the geographic area, genetics, and environmental factors (climate and dietary habits). ${ }^{(5)}$

Spontaneous elimination of stones was found to occur in $46 \%$ of patients, with an average size of $5.3 \mathrm{~mm}$. The probability of spontaneous elimination is reduced if the stone is larger than 3-4 mm in diameter. ${ }^{(12)}$ It was noted that children older than 5 years were more prone to spontaneous evacuation. ${ }^{(13)}$ If the stone has passed spontaneously, stone chemical analysis will identify specific stone composition and lead to further workup. Struvite stone is associated with infection; a urine sample for culture must be taken. Urinary cystine should be measured if a cystine stone is found. Calcium oxalate or calcium phosphate stones must be followed by complete metabolic evaluation, which for pediatric nephrolithiasis comprises urinalysis, urine culture, spot urine to measure calcium-to-creatinine ratio, serum chemistries (electrolytes, uric acid, creatinine, calcium, phosphorus, bicarbonate, and magnesium), and 24-hour urine ( $\mathrm{pH}$, volume, calcium, oxalate, uric acid, cystine, citrate, magnesium, sodium, phosphorus, and potassium adjusted for urinary creatinine). Analysis of our patient's stone revealed calcium, oxalate, phosphate, and cystine. The most probable main component of this patient's stone is calcium oxalate and/or calcium phosphate, which is consistent with data from most of the literature. Approximately $60-90 \%$ of patients with urolithiasis form calcium stones, most of which are composed primarily of calcium oxalate or, less often, calcium phosphate. The majority of calcium stones form in otherwise healthy individuals without systemic disease. ${ }^{(6,14)}$ Struvite constitutes $1-18 \%$ of the stones in developed countries; uric acid constitutes $5-10 \%$, cysteine $1-5 \%$, and mixed stones $4 \%{ }^{(6)}$

Based on metabolic evaluation, this patient had hypercalciuria with normal serum calcium and normal renal function (normocalcemic hypercalciuria). ${ }^{(15)}$ Hypercalciuria is considered idiopathic if the serum calcium level is normal and known possible causes of normocalemic hypercalciuria can be excluded. Some differentials of normocalcemic hypercalciuria are idiopathic hypercalciuria, hyperparathyroidism, furosemide or corticosteroid therapy, immobilization, Bartter syndrome, Syberth syndrome, early vitamin D toxicity, limb fracture, thyrotoxicosis, and distal renal tubular acidosis (RTA). ${ }^{(16)}$ Idiopathic hypercalciuria (IH) is a common metabolic abnormality in children. The gene responsible for familial IH has not been identified, but appears to be transmitted in an autosomal dominant manner. ${ }^{(12)}$ The normal upper limit for calcium excretion in children is generally considered to be $4 \mathrm{mg} / \mathrm{kg} / \mathrm{day}$ and is best measured from 24-hour urine collection, ${ }^{(15)}$ which is recommended to exclude diurnal fluctuations related to intake of food and beverages. ${ }^{(9)}$ The urinary calcium-to-creatinine ratio ( $\mathrm{UCa} / \mathrm{UCr}$ ratio) can be used as a screening test if 24-hour urine collection cannot be done. A ratio exceeding 0.21 in random voided urine can be defined as hypercalciuria. ${ }^{(15)}$

In this patient, some etiology differentials for hypercalciuria can be excluded through the history and physical examination. The patient did not have a history of furosemide or corticosteroid therapy or immobilization, nor a family history of hereditary hypercalciuria, or limb fracture. The history and physical examination did not reveal signs of thyrotoxicosis or sarcoidosis. RTA that is associated with renal stones is distal RTA (type I), having the clinical findings of hyperchloremic acidosis, minimum urine $\mathrm{pH}$ of $>5.5$, and low to normal plasma potassium, which were absent in this patient. Normal serum electrolytes ruled out renal tubular acidosis and Bartter syndrome. ${ }^{(15)}$ Therefore, the probability of idiopathic hypercalciuria can be declared. 
Idiopathic hypercalciuria can have a familial predisposition or can occur in sporadic form. In the familial form, the inheritance is compatible with autosomal dominant transmission. ${ }^{(6)}$ Idioptahic hypercalciuria results from either a combination of increased gastrointestinal calcium absorption from a direct increase in calcium absorption or through excess 1,25dihydroxyvitamin D-mediated calcium absorption; decreased renal absorption of either calcium or phosphorus; or enhanced bone resorption. Formal assessment for subtypes of hypercalciuria in children on a regular basis is not mandatory, but it can be utilized as a reasonable framework to methodically approach an evaluation of a medically complex child with hypercalciuria. ${ }^{(17)}$ In children, calcium intake should not be limited. In fact, low calcium diets have been associated with increased risk of nephrolithiasis while high dietary calcium intake has been associated with decreased risk of stones in adults. This is likely due to the ability of dietary calcium to decrease the intestinal absorption of oxalate. ${ }^{(18)}$

The risk of nephrolithiasis increases progressively with the greater levels of IH. In $85 \%$ of children with $\mathrm{IH}$, renal microlithiasis has been reported in follow-up US. Most cases of IH have non-specific symptoms and are commonly associated with symptoms of urolithiasis. IH can manifest as lower urinary tract signs and symptoms probably due to microcrystallization causing injury to the urinary tract epithelia. The typical presentations of urolithiasis in children are (1) intense colicky abdominal pain, (2) gross hematuria with or without pain, (3) urinary infection, (4) asymptomatic stones, or (5) persistent microscopic hematuria, which consists of 5 or more RBCs per hpf in 3 of 3 consecutive urine specimens obtained at least 1 week apart. ${ }^{(16)}$ Gross hematuria in IH is often transient, or persists for several days. ${ }^{(15)}$ This patient's symptomstransient macroscopic hematuria and recurrent UTI-were associated with the urolithiasis.

Considering the history of recurrent UTI in this patient, a prophylactic antibiotic was indicated to prevent reinfection. Prophylactic antibiotic using either the sulfamethoxazoletrimethoprim or nitrofurantoin at one third of the normal therapeutic dose once a day is often effective. Prophylaxis with amoxicillin or cephalexin also may be effective, but the risk of breakthrough UTI is higher because bacterial resistance may be induced. ${ }^{(3)}$ This patient's condition warrants urgent urologic surgery consultation since the stone was associated with urinary obstruction and recurrent pyelonephritis. Hypercalciuria in this patient is probably idiopathic, but the treatment for the hypercalciuric condition has to be done to prevent recurrent stone formation.

For long-term management or prevention of recurrence, clinical treatment is needed. Treatment aims to reduce or prevent the emergence of new stones or prevent existing ones to increase in size, thus reducing morbidity and the need for surgical intervention. The clinical treatment includes nonpharmacological and pharmacological treatment. The non-pharmacological approach, i.e. increasing the fluid intake and dietary adequacy, is the most important approach. ${ }^{(14)} \mathrm{An}$ increased fluid intake under close monitoring is critical in preventing super saturation of the urine. Dietary sodium restriction along with a high-potassium, low-oxalate diet is recommended.(19) Pharmacotherapy is recommended if the fluid and dietary approach has failed. The most common drug used is a thiazide diuretic, such as hydrochlorothiazide $(1-2 \mathrm{mg} / \mathrm{kg} /$ day $) .{ }^{(12)} \mathrm{In}$ addition to fluid intake and diet modification, thiazide diuretics remain the major therapy for calcium stones associated with IH. Thiazide drugs reduce calcium excretion by increasing calcium absorption in the proximal tubule and stimulating calcium reabsorption in the distal tubule. ${ }^{(14)}$

Between $25-50 \%$ of children with nephrolithiasis are estimated to undergo surgical management. The most common options are extracorporeal shockwave lithotripsy (ESWL), retrograde intrarenal surgery (RIRS), and percutaneous nephrolithotomy (PCNL). Open surgery is rarely needed. ${ }^{(19)}$ ESWL is 
recommended for small upper tract stones, in children without cystinuria or stones secondary to obstructive uropathy. Current guidelines recommend ESWL and PCNL as first line treatment for renal stones $<2$ and $>2 \mathrm{~cm}$ in diameter respectively. However, RIRS is gaining more acceptance as a first line alternative option for renal stones up to $2 \mathrm{~cm}$ or more. RIRS can reduce complications related to PCNL, namely urinary sepsis and bleeding. ${ }^{(20)}$

Long-term complications of pediatric nephrolithiasis are hydronephrosis, recurrent urinary infection associated with renal scarring, renal failure, anemia, and hypertension. Previously, renal scarring has been reported to occur in $8 \%$ of children with urolithiasis. ${ }^{(5)}$ The prognosis for nephrolithiasis depends on the primary diagnosis and adherence to therapy, but recurrence rates generally are high when the condition is left untreated. Recurrence of pediatric urolithiasis ranges from $6.5-44 \%$ with a mean interval of 3-6 years. Without follow-up and medical intervention, recurrence rates have been reported as high as $50 \%$ within $5-6$ years. ${ }^{(8)}$

A limitation of this report was the incomplete illustration of the clinical course of the patient, i.e. the long-term follow-up to identify recurrences and complications. We also did not do further investigations in the family to identify a possible familial cause.

\section{CONCLUSIONS}

Hypercalciuria is the most common cause of stones in children, in which most of them are idiopathic. Treatment of the stones and the underlying hypercalciuria is important to prevent recurrent stone formation and long-term complications. It should always be kept in mind that the renal stone is the sign of the disease, but not the disease it self.

\section{CONFLICT OF INTERESTS}

Competing interests: no relevant disclosures.

\section{ACKNOWLEDGMENT}

The authors thank the postgraduate students on pediatric internship, Visakha Irawan and Maria Patricia, for helping with the data collection.

\section{CONTRIBUTORS}

NY contributed to data collection, manuscript drafting, and analysis of the case. I contributed to analysis of the case and revision of manuscript. Both authors read and approved the final manuscript.

\section{REFERENCES}

1. Kokorowski PJ, Hubert K, Nelson CP. Evaluation of pediatric nephrolithiasis. Indian J Urol 2010; 26:531-5.

2. Sas DJ. An update on the changing epidemiology and metabolic risk factors in pediatric kidney stone disease. Clin J Am Soc Nephrol 2011;6: 2062-8.

3. Elder JS. Urinary tract infections. In: Kliegman R, Stanton B, JS Geme, Schor N, editors. Nelson Textbook of Pediatrics, $19^{\text {th }}$ ed. Philadelphia: Elsevier;2011.p.1785-9.

4. Valentini RP, Lakshmanan Y. Nephrolithiasis in children. Adv Chronic Kidney Dis 2011;18:3705. doi: 10.1053/j.ackd.2011.07.002.

5. Elmaci AM, Ece A, Akin F. Clinical characteristics and metabolic abnormalities in preschool-age children with urolithiasis in southeast Anatolia. J Ped Urol 2013;xx:1-5.

6. Sharma AP, Giller G. Epidemiology of pediatric urolithiasis. Indian J Urol 2010;26:51-22.

7. Habbig S, Beck BB, Hoppe B. Nephrocalcinosis and urolithiasis in children. Kidney Int 2011; 80:1278-91. doi: 10.1038/ki.2011.336.

8. Koyuncu H, Yencilek F, Ertuhan S, et al. Clincal course of pediatric urolithiasis: follow-up data in a long-term basis. Int Urol Nephrol 2011; 43:7-13.

9. Hoppe B, Kemper MJ. Diagnostic examination of the child with urolithiasis or nephrocalcinosis. Pediatr Nephrol 2010;25:403-413.

10. Asl AS, Maleknejad S. Pediatric urolithiasis: an experience of a single center. IKJD 2011;5:30913.

11. Alpay H, Ozen A, Gokce I, et al. Clinical and metabolic features of urolithiasis and 
microlithiasis in children. Pediatr Nephrol 2009; 24:2203e9.

12. Copelovitch L. Urolithiasis in children. Pediatr Clin N Am 2012;59:881-96.

13. Milošević D, Batinić D, Turudić D, et al. Demographic characteristics and metabolic risk factors in Croatian children with urolithiasis. Eur J Pediatr 2014;173:353-9.

14. Amancio L, Fedrizzi M, Bresolin NL, et al. Pediatric urolithiasis: experience at a tertiary care pediatric hospital. J Bras Nefrol 2016;38:90-8.

15. Gillespie RS. Stapelton FB. Nephrolithiasis in children. Pediatr Rev 2010;25:131-40.

16. Esfhani ST, Madani A, Siadati AA, et al. Prevalence and symptoms of idiopathic hypercalciuria in primary school children of Tehran. Iran J Pediatr 2007;17:353-8.
17. Srivastava T, Schwaderer A. Diagnosis and management of hypercalciuria in children. Curr Opin Pediatr 2009;21:214-9.

18. Jackson EC, Reeber MA. Urolithiasis in children: treatment and prevention. Curr Treat Options Peds 2016;2:10-22. doi: 10.1007/ s40746-016-0041-0.

19. Chu DI, Tasian GE, Copelovitch L. Pediatric kidney stones - avoidance and treatment. Curr Treat Options Peds 2016;2:104-11.

20. Salerno A, Nappo SG, Matarazzo E, et al. Treatment of pediatric renal stones in a Western country: a changing pattern. J Pediatr Surg 2013; 48:835-9. 